\title{
List of reviewers
}

The Editors would like to thank the following individuals who kindly agreed to serve as referees for the Journal between October 1, 2015 and August 31, 2016.

$\begin{array}{lll}\text { Sacit Hadi Akdede } & \text { John Galbraith } & \text { Luc Renneboog } \\ \text { Peter Alhadeff } & \text { David Giles } & \text { Luciano Rossoni } \\ \text { Hasan Bakhshi } & \text { V. Ginsburgh } & \text { Michael Rushton } \\ \text { Alan Beggs } & \text { Christian Handke } & \text { F. M. Scherer } \\ \text { Francoise Benhamou } & \text { Thorsten Hennig-Thurau } & \text { Antonello Scorcu } \\ \text { Trine Bille } & \text { Luis César Herrero- } & \text { B. A. Seaman } \\ \text { Karol Borowiecki } & \text { Prieto } & \text { J. D. Snowball } \\ \text { Stephen Boyle } & \text { Douglas Hodgson } & \text { Christophe Spaenjers } \\ \text { Dmitri Byzalov } & \text { Magnus Hoffmann } & \text { Jessica Stahl } \\ \text { Cedric Ceulemans } & \text { Michael Hutter } & \text { Elena Stapanova } \\ \text { Lesley Chiou } & \text { Stephanie Jozefowicz } & \text { Jacques-Francois Thisse } \\ \text { Darlene Chisholm } & \text { Stan Liebowitz } & \text { M. Kathleen Thomas } \\ \text { Annalisa Cicerchia } & \text { Judy Ma } & \text { Rex Thompson } \\ \text { Alan Collins } & \text { Francisco Marco- } & \text { David Throsby } \\ \text { Joe Cox } & \text { Serrano } & \text { Ruth Towse } \\ \text { Dakshina De Silva } & \text { Isidoro Mazza } & \text { Gautam Vora } \\ \text { S. Gerhard Dijkstra } & \text { Jordi Mckenzie } & \text { Patrick Waelbroeck } \\ \text { Bulent Dogru } & \text { Anna Mignosa } & \text { W. D. Walls } \\ \text { Robert Ekelund } & \text { Francois Moreau } & \text { Miao Wang } \\ \text { Federico Etro } & \text { Tylor Orme } & \text { Wei-jen Wen } \\ \text { Nathalie Fabry } & \text { Alessandra Pelloni } & \text { Heike Wetzel } \\ \text { Guido Ferilli } & \text { Rachel Pownall } & \text { Daniel Wheatley } \\ \text { Victor Fernandez- } & \text { Oscar Quiros } & \text { Hyunjoo Yang } \\ \text { Blanco } & \text { S. Abraham (Avri) Ravid } & \\ \text { Darren Filson } & \text { Imke Reimers } & \end{array}$

